\title{
A New Einstein Cross Gravitational Lens of a Lyman-break Galaxy
}

\author{
Daniela Bettoni $^{1}$ (10), Renato Falomo ${ }^{1}$ (1) , Riccardo Scarpa $^{2,3}$ (1) , Mattia Negrello $^{4}$, Alessando Omizzolo ${ }^{1,5}$, \\ Romano L. M. Corradi ${ }^{2,3}$ (D), Daniel Reverte ${ }^{2,3}$, and Benedetta Vulcani ${ }^{1}$ (D) \\ ${ }^{1}$ INAF-Osservatorio Astronomico di Padova, Vicolo Osservatorio 5 I-35122 Padova, Italy; daniela.bettoni@inaf.it \\ ${ }^{2}$ GRANTECAN, Cuesta de San Jos s/n, E-38712, Brea Baja, La Palma, Spain \\ ${ }^{3}$ Instituto de Astrofisica de Canarias, Vía Lactea s/n, E-38200, La Laguna, Tenerife, Spain \\ ${ }^{4}$ School of Physics and Astronomy, Cardiff University, The Parade, Cardiff CF24 3AA, UK \\ ${ }^{5}$ Vatican Observatory, Vatican City State, Vatican City, Italy \\ Received 2019 February 12; revised 2019 February 27; accepted 2019 February 27; published 2019 March 11
}

\begin{abstract}
We report the study of an "Einstein Cross" configuration first identified in a set of HST images by Cerny et al. Deep spectroscopic observations obtained at the Spanish $10.4 \mathrm{~m}$ Gran Telescopio Canarias telescope, allowed us to demonstrate the lens nature of the system, that consists of a Lyman-break galaxy (LBG), not a quasi-stellar object as is usually the case, at $z=3.03$ lensed by a galaxy at $z=0.556$. Combining the new spectroscopy with the archival $H S T$ data, it turns out that the lens is an elliptical galaxy with $M_{V}=-21.0$, effective radius $2.8 \mathrm{kpc}$, and stellar velocity dispersion $\sigma=208 \pm 39 \mathrm{~km} \mathrm{~s}^{-1}$. The source is an LBG with Ly $\alpha$ luminosity $\sim L^{*}$ at that redshift. From the modeling of the system, performed by assuming a singular isothermal ellipsoid (SIE) with external shear, we estimate that the flux source is magnified about 4.5 times, and the velocity dispersion of the lens is $\sigma_{\mathrm{SIE}}=197.9_{-1.3}^{+2.6} \mathrm{~km} \mathrm{~s}^{-1}$, in good agreement with the value derived spectroscopically. This is the second case known of an Einstein cross of an LBG.
\end{abstract}

Key words: galaxies: elliptical and lenticular, $\mathrm{cD}$ - galaxies: high-redshift - gravitational lensing: strong techniques: spectroscopic

\section{Introduction}

Gravitational lenses represent one of the most powerful tools to probe the properties of distant galaxies and the cosmological parameters. Strong gravitational lensing produces multiple images of distant sources that have their lines of sight very close to foreground massive objects (see Treu 2010, for a review). In addition, the special case of image splitting of distant quasars can provide the direct measurement of the Hubble constant from correlated flux variability (Treu \& Koopmans 2002; Suyu et al. 2014).

Accurate lens modeling can precisely probe the density profile of galaxies at cosmological distances, specifically the mass enclosed within the Einstein radius and the mean local density slope within it. Combining lensing and dynamics allows the central dark matter profile to be robustly inferred (e.g., Treu \& Koopmans 2004). All these facts make this kind of configuration a fantastic laboratory for the study of the universe.

Of particular interest is the detection of quadruple images of lensed quasi-stellar object (QSO) in the shape of an Einstein Cross (see e.g., Wisotzki et al. 2002; Morgan et al. 2004 for first discoveries). However, these optical structures are rare on the sky (Oguri \& Marshall 2010) as they require a very close alignment of quasars with foreground massive galaxies. Various large-area sky surveys are planned in the near future to increase the number of such systems (e.g., Schechter et al. 2017; Williams et al. 2017, 2018; Agnello et al. 2018), and recently the $H_{o}$ Lenses in COSMO-GRAILs Wellspring (HOLiCOW) program (Bonvin et al. 2017; Suyu et al. 2017) listed the five best lensed quasars discovered to date showing an Einstein Cross structure.

During a search for high- $z$ galaxies from the Reionization Lensing Cluster Survey (RELICS) of the Hubble Treasury Program (Salmon et al. 2017), a possible new Einstein Cross configuration around a galaxy located at $\alpha=22: 11: 41.99$, $\delta=03: 50: 52.3$ was discovered by Cerny et al. (2018; see Figure 1). Later on, during a search for stripped galaxies in the Hubble Space Telescope (HST) images from the RELICS project, the serendipitous re-discovery of this object by one of us (A. O.) led to the observations presented in this Letter. The system, hereafter called J2211-0350, is sitting $\sim 90$ arcsec southwest from the core of the cluster RXC J2211.7-0349 $(z=0.397)$ and, based on the redder color of the alleged lens galaxy, it was argued that the lens had to be well behind the nearby cluster. The system is composed by an early-type red galaxy surrounded by four blue objects that are arranged in the shape of a "Latin Cross" around the central galaxy. In Table 1 we report the magnitudes and the relative positions of the image components. Data are from the RELICS catalogs except for the component $\mathrm{D}$ that we measured directly on the HST images.

Prompted by this discovery, we report here the results of optical spectroscopy of the system that allows us to confirm its lensing nature and measure the redshift of both the lens and the source. We adopt the concordance cosmology and assume $H_{0}=70 \mathrm{~km} \mathrm{~s}^{-1} \mathrm{Mpc}^{-1}, \Omega_{m}=0.3$, and $\Omega_{\Lambda}=0.7$.

\section{Observations and Data Analysis}

Spectroscopic observations were collected on 2018 December 1 at the $10.4 \mathrm{~m}$ Gran Telescopio Canarias, located at the Roque de Los Muchachos observatory, La Palma (Spain). We used the optical spectrograph OSIRIS (Cepa et al. 2003) with the grism R1000B covering the spectral range $4100-7500 \AA$, and a slit width of 1.0 arcsec. This configuration yields an effective spectral resolution of $R \sim 600$.

Figure 1 shows an HST image of the target with superimposed the position of the slit, located so to observe both the lensing galaxy and the three brighter images of the source at 


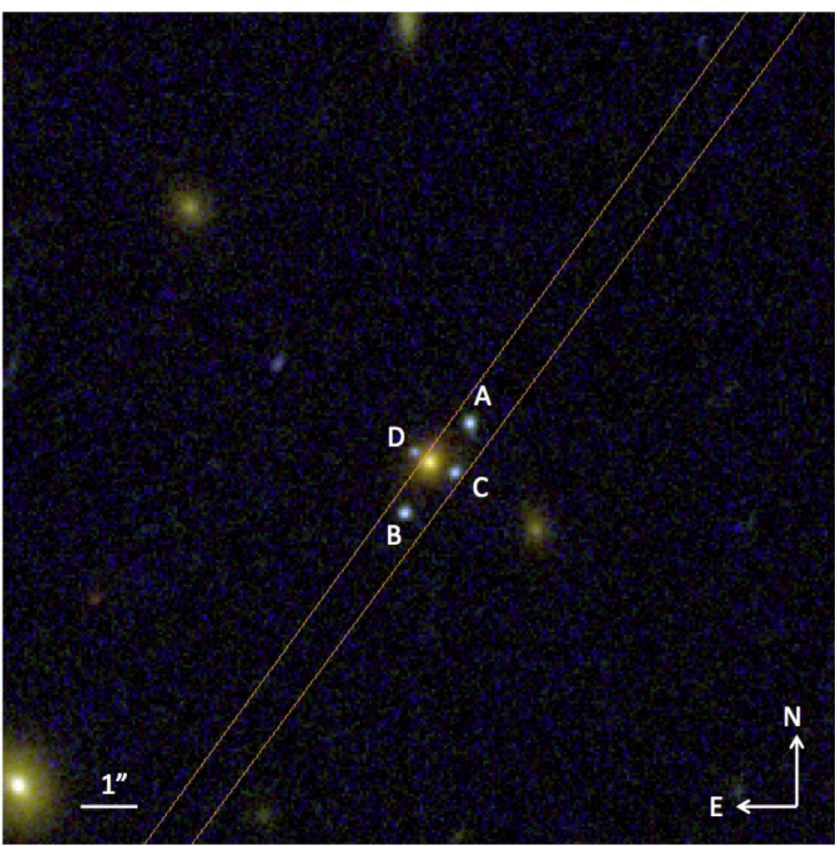

Figure 1. HST color image of the new Einstein Cross J2211-0350 in the field of the cluster RXC J2211.7-0349. The image is a combination of WFC3/IR (F160W) in red, ACS image $(\mathrm{F} 814 \mathrm{~W})$ in green, and $(\mathrm{F} 435 \mathrm{~W})$ in blue. The orange lines represent the position of the 1 arcsec slit used for the spectroscopic observations. Both the lens galaxy and three lensed targets are observed at once.

Table 1

Position and Measured Magnitudes

\begin{tabular}{lccccc}
\hline \hline Id. & $\begin{array}{c}\Delta \alpha \\
\operatorname{arcsec}^{\mathrm{a}}\end{array}$ & $\begin{array}{c}\Delta \delta \\
\operatorname{arcsec}^{\mathrm{a}}\end{array}$ & $\begin{array}{c}\text { F435W } \\
\mathrm{AB}\end{array}$ & $\begin{array}{c}\text { F606W } \\
\mathrm{AB}\end{array}$ & $\begin{array}{c}\text { F814W } \\
\mathrm{AB}\end{array}$ \\
\hline Gal & \multicolumn{1}{c}{0} & 0 & $24.61 \pm 0.08$ & $22.83 \pm 0.01$ & $21.59 \pm 0.01$ \\
$\mathrm{~A}$ & 0.826 & -0.756 & $24.80 \pm 0.06$ & $24.21 \pm 0.02$ & $24.19 \pm 0.03$ \\
$\mathrm{~B}$ & -0.481 & 1.021 & $24.88 \pm 0.06$ & $24.38 \pm 0.02$ & $24.25 \pm 0.03$ \\
$\mathrm{C}$ & 0.517 & 0.210 & $25.24 \pm 0.06$ & $24.69 \pm 0.02$ & $24.54 \pm 0.03$ \\
$\mathrm{D}$ & -0.324 & -0.216 & $25.7 \pm 0.10$ & $25.20 \pm 0.15$ & $25.11 \pm 0.10$ \\
\hline
\end{tabular}

Notes. R.A. (2000) = 221141.97 decl. $(2000)=-035052.0$.

a Positions relative to the lensing galaxy center.

once. Only source D is outside the slit. Three independent exposures of $1800 \mathrm{~s}$ were obtained under photometric conditions and good atmospheric seeing ( 0.8 arcsec).

Standard IRAF ${ }^{6}$ tools were adopted for the data reduction. Bias subtraction, flat field correction, image alignment, and combination were performed. Cosmic rays were cleaned by combining the three independent exposures and using the crreject algorithm. The spectra were then calibrated in wavelength with $\sim 0.2 \AA$ accuracy. Data of a spectrophotometric standard star observed on the same night were used to perform a relative flux calibration of the spectrum. HST photometry on filter F606W was then used to achieve absolute flux calibration of the spectrum (see Table 1).

The final 2D spectrum is characterized by the strong emission from three blobs coincident with the spatial location in the slit of the three lensed sources (see Figures 2 and 3). This leaves no doubt about the nature of this source. The emission,

\footnotetext{
6 IRAF is distributed by the National Optical Astronomy Observatory, which is operated by the Association of Universities for Research in Astronomy (AURA) under cooperative agreement with the National Science Foundation.
}

centered at $\lambda=4904 \AA$, is identified as the $\operatorname{Ly} \alpha$ at $z=3.03$. No other clear emission lines are visible (see Figure 4) implying that the lensed source is a Lyman-break galaxy (LBG) rather than a QSO. The identification of this line is secure as there are no other emission lines and its profile shows a classic, asymmetric, blue self-absorbed morphology that is typical of Ly $\alpha$ emission (Jones et al. 2012).

\section{Results}

The flux and shape of the Ly $\alpha$ emission for the three blobs are very similar, the observed FWHM for this line is $10-12 \AA$ corresponding to a velocity of $\sim 1200 \mathrm{~km} \mathrm{~s}^{-1}$ at rest frame (Figures 3 and 5).

The observed flux is $2.4 \times 10^{-16} \mathrm{erg} \mathrm{cm}^{-2} \mathrm{~s}^{-1}$. Including the contribution of source $\mathrm{D}$ (estimated from the HST photometry), we derive a total observed line luminosity of $L(\operatorname{Ly} \alpha)=2.5 \times 10^{43} \mathrm{erg} \mathrm{s}^{-1}$.

The red part of the spectrum is dominated by the signal from the lens galaxy. Using the pPXF (Cappellari \& Emsellem 2004) IDL routines we measured the stellar velocity dispersion. The $\mathrm{Ca}$ II $\mathrm{H}$ and $\mathrm{K}$ absorption lines were fitted at $z=0.556$ to estimate the galaxy velocity dispersion. We used the library of single stellar population spectra from Vazdekis et al. (2012) properly convolved with the instrumental resolution to fit the lines' profile. The best fit corresponds to a velocity dispersion $\sigma=208 \pm 39 \mathrm{~km} \mathrm{~s}^{-1}$. These results confirm the photometric redshift found by Cerny et al. (2018) that the galaxy is not a member of the cluster RXC J2211.7-0349.

The ACS $+\mathrm{F} 814 \mathrm{~W}$ image was used to derive the properties of the lensing galaxy. After properly masking the four images of the source, we modeled the lens galaxy with a Sérsic law. The data were deconvolved using as point-spread function (PSF) an unsaturated and isolated star present in the field. We found that the lens galaxy is well fitted by a model with $n=5$ and effective radius $r_{e}=0.44$ arcsec corresponding to $R_{e} \sim 2.8 \mathrm{kpc}$. The absolute magnitude of the lens galaxy $(z=0.556)$ corresponds to $M_{V} \sim-21.0$ (taking into account $k$-correction). The mass of the lens galaxy estimated from the measured $\sigma$ and $R_{e}$ is $M_{\mathrm{gal}}=4.5 \times 10^{10} M_{\odot}$.

\subsection{Lens Modeling}

We performed the lens modeling of the system by using the code by Enia et al. (2018), which implements the regularized semi-linear inversion formalism with adaptive pixel scale (Warren \& Dye 2003; Suyu et al. 2006; Nightingale \& Dye 2015, and references therein).

The source plane (SP; i.e., the plane orthogonal to the line of sight of the observer to the deflector, containing the background source) is gridded into pixels whose values represent the source surface brightness counts and are treated as free parameters. This approach avoids any a priori analytic assumption on the surface brightness of the background source. The size of the pixels adapts to the magnification pattern, being smaller closer to the regions of increasing magnification, to ensure a uniform signal-to-noise ratio $(\mathrm{S} / \mathrm{N})$ across the reconstructed source and to fully exploit the increase in spatial resolution in highly magnified regions provided by gravitational lensing. For a fixed mass model of the deflector (or lens), the SP is mapped into the image plane (IP; i.e., the plane containing the deflector and orthogonal to the line of sight of the observer to the deflector), then convolved with the PSF, and 


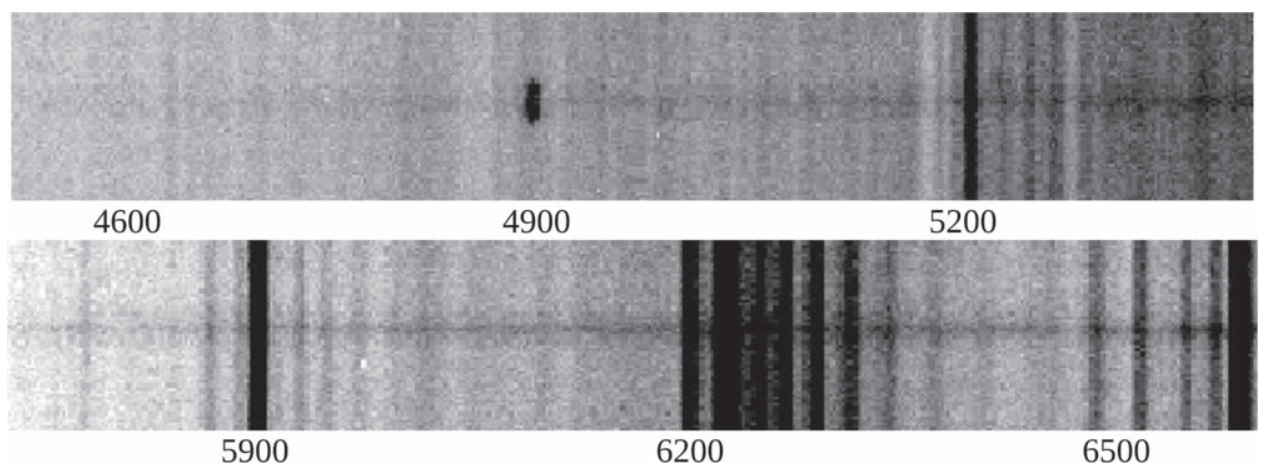

Figure 2. The 2D optical spectrum of the Einstein Cross J2211-0350 (see also Figure 3). Superimposed to the faint continuum is a prominent emission line at $\sim 4900 \AA$ Adentified as Ly $\alpha$.

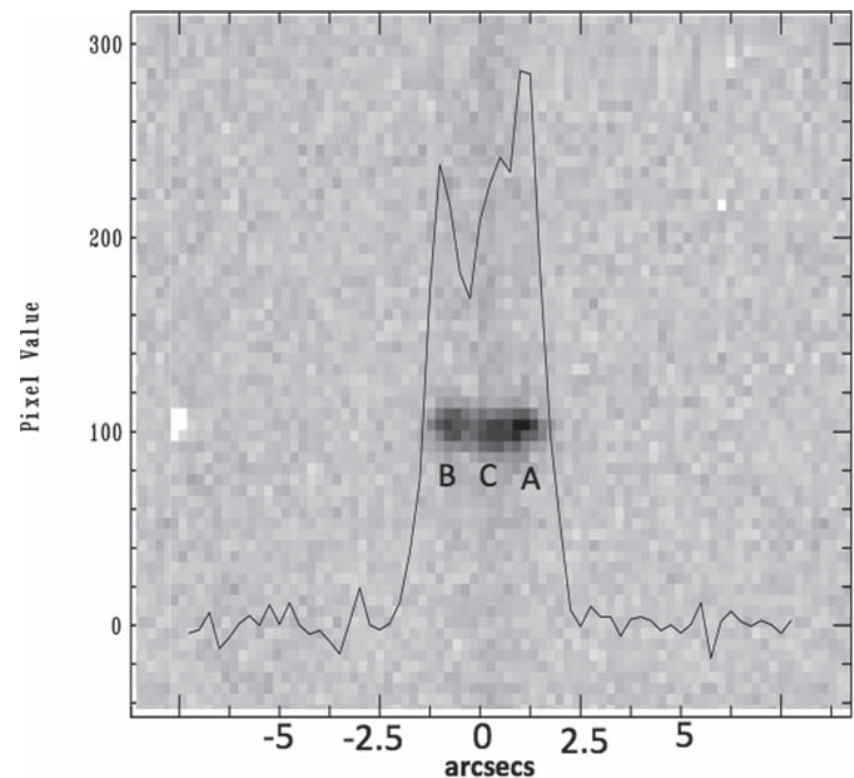

Figure 3. Enlargement of the region around the Ly $\alpha$. The Ly $\alpha$ emission of the three lensed images (A, B, and C) is clearly resolved. Note the slight offset of component $\mathrm{C}$ with respect to $\mathrm{A}$ and $\mathrm{B}$ due to the different position inside the slit (see also Figure 1). The run of the flux along the spatial direction is also shown (solid line).

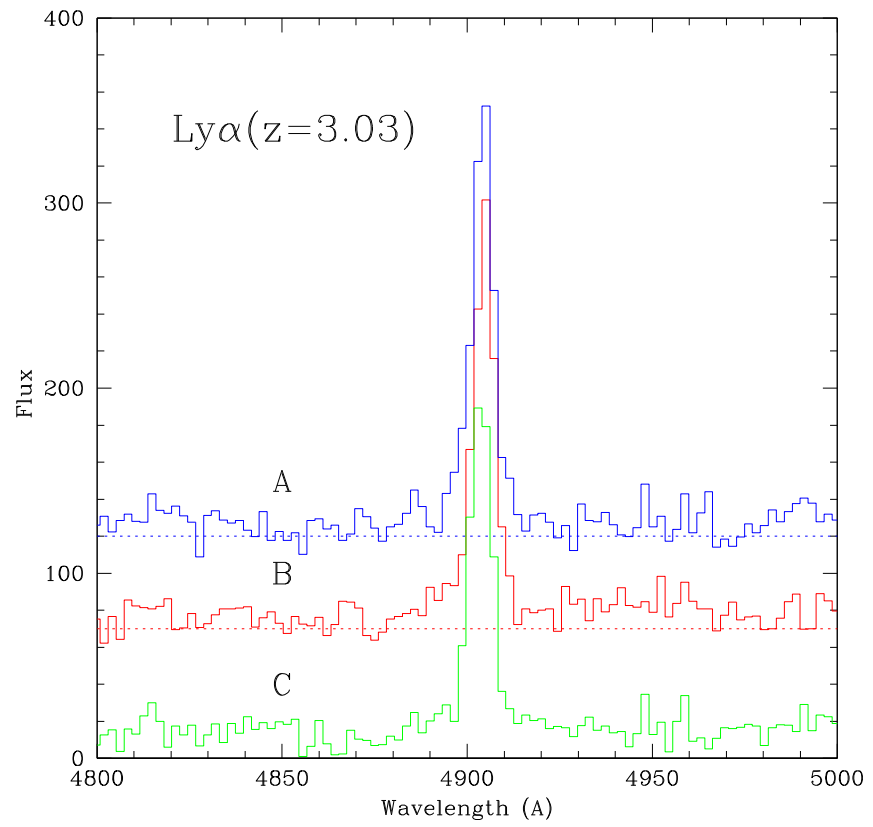

Figure 5. Ly $\alpha$ region of optical spectrum of the three images of the Einstein Cross shifted of an arbitrary quantity to viewing purposes. The peak is labeled "A" in Figure 3.

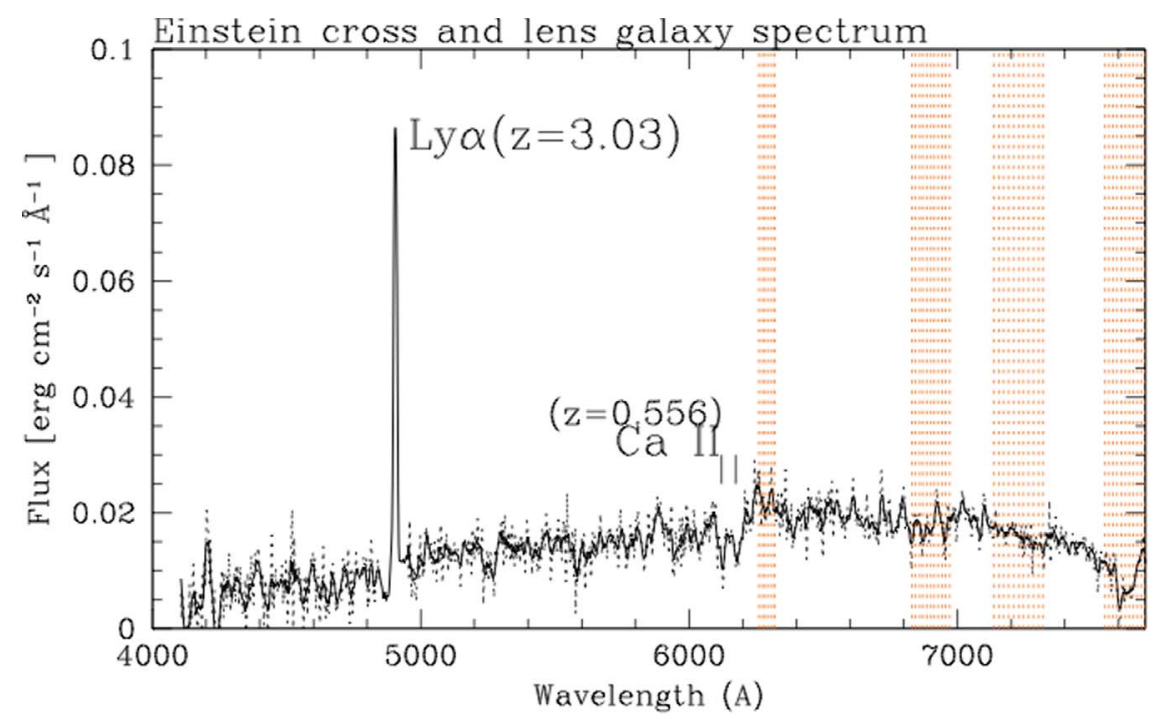

Figure 4. Optical spectrum of the Einstein Cross (observed frame). This 1D spectrum was obtained by integrating the flux of both the lens galaxy and three lensed images (see also Figure 2). A prominent Ly $\alpha$ emission is visible at $z=3.03$ due to the lensed source while stellar absorption lines at $z=0.556$ are due to the lensing galaxy. The regions affected by telluric absorptions are marked (orange vertical lines). 

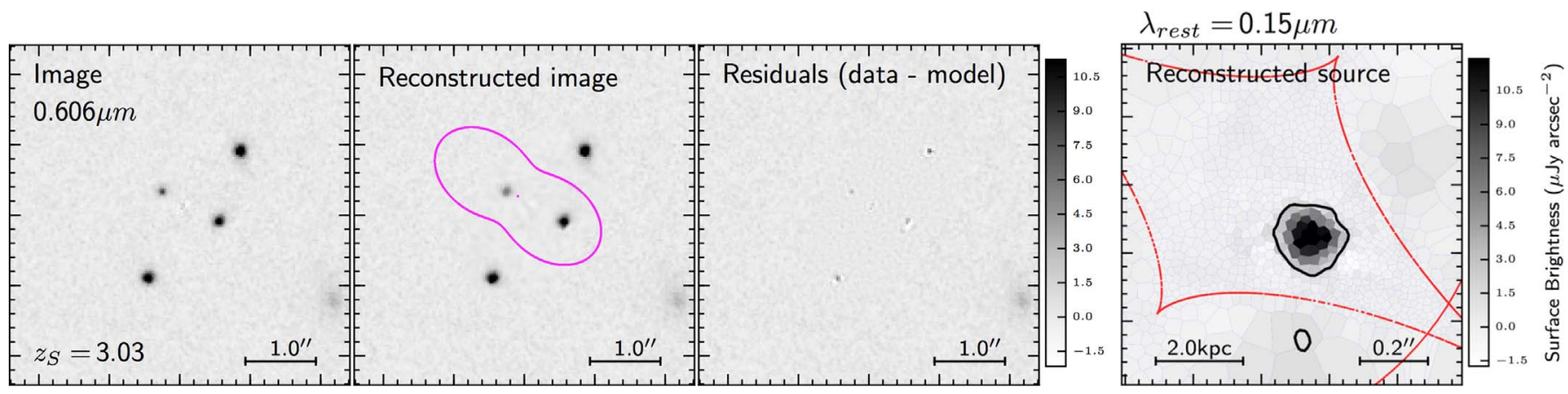

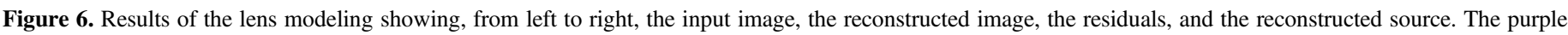
curve in the second panel from the left is the tangential critical line, while the red curves in the rightmost panel are the caustics.

finally compared to the observed image. In order to avoid unphysical solutions, in with the reconstructed source presenting severe discontinuities and pixel-to-pixel variations, a regularization term is added to the merit function. The weight of the regularization term is calculated via Bayesian analysis, according to Suyu et al. (2006).

The mass distribution of the lens is modeled as a singular isothermal ellipsoid (SIE), described by the following parameters: the Einstein radius $\left(\theta_{\mathrm{E}}\right)$, the position of the lens centroid $\left(x_{\mathrm{L}}, y_{\mathrm{L}}\right)$, the minor-to-major axis ratio $\left(q_{\mathrm{L}}\right)$, and the orientation angle $\left(\theta_{\mathrm{L}} ;\right.$ counter-clockwise from west). Although the object is at the edge of the nearby cluster, to model the lens an external shear is included in the model. It is described by the shear strength $(\gamma)$ and the shear angle $\left(\theta_{\gamma}\right.$; counter-clockwise from west). The search for the best-fitting parameters of the lens is done using the EMCEE code (Foreman-Mackey et al. 2013), which implements the Markov Chain Monte Carlo technique to sample the posterior probability density function of the model parameters.

The magnification factor, $\mu$, is calculated as the ratio between the total flux density of the sources, as measured in the SP within the region of $\mathrm{S} / \mathrm{N} \geqslant 3$, and the flux density of the corresponding image in the IP. The uncertainty on the magnification factor is derived by computing $\mu 1000$ times, perturbing each time the lens model parameters around their best-fitting values.

The modeling is carried out on the reduced ACS + F606w image, with a pixel scale of 0 ". 06 . A noise map is constructed from the provided weight map and the PSF is obtained by median combining three unsaturated stars in the vicinity of the target. The lens is subtracted from the image after fitting its light profile with GALFIT (Peng et al. 2002).

The best-fitting SIE model has $\theta_{\mathrm{E}}=0.76_{-0.01}^{+0.02}$ arcsec, $q_{\mathrm{L}}=0.66_{-0.06}^{+0.04}$, and $\theta_{\mathrm{L}}=-35.6_{-0.8}^{+0.6}$ degrees, with an external shear of strength $\gamma=0.31_{-0.02}^{+0.01}$ and angle $\theta_{\gamma}=-34.9_{-0.7}^{+0.6}$ degrees. The estimated Einstein radius can be converted into a velocity dispersion, $\sigma_{\mathrm{SIE}}$, using the relation

$$
\theta_{\mathrm{E}}=4 \pi\left(\frac{\sigma_{\mathrm{SIE}}}{c}\right)^{2} \frac{D_{\mathrm{LS}}}{D_{\mathrm{S}}}
$$

where $D_{\mathrm{LS}}$ and $D_{\mathrm{S}}$ are the angular-diameter distances from lens to source and observer to source, respectively. We find $\sigma_{\mathrm{SIE}}=197.9_{-1.3}^{+2.6} \mathrm{~km} \mathrm{~s}^{-1}$, which is in good agreement with the value derived from the line profile.

The results of the lens modeling are shown in Figure 6. The reconstructed source, in the rightmost panel, is compact. This result is not surprising, as there is no evidence of extended structure in the lensed images. The estimated magnification factor is $\mu=4.5_{-0.8}^{+1.0}$.

\section{Summary and Conclusions}

We have presented the spectroscopic confirmation of a new gravitational lens J2211-0350 with an Einstein Cross configuration discovered while inspecting RELICS images. The lens is an elliptical galaxy $\left(M_{V} \sim-21\right)$ at $z=0.556$, while the lensed source is an LBG at $z=3.03$. Modeling of the lens shows that the Einstein Cross is well reproduced by an SIE model with an Einstein ring of 0.76 arcsec and that includes the shear effect due to the foreground massive low-redshift cluster of galaxies. The lensed source is magnified by a factor 4.5 . This gravitational lens is similar to the case reported by Bolton et al. (2006) for J1011+0143. This is the second case of an Einstein Cross gravitational lens produced by a distant LBG. The intrinsic Ly $\alpha$ luminosity, taking into account the magnification factor, is $L(\operatorname{Ly} \alpha)=5 \times 10^{42} \mathrm{erg} \mathrm{s}^{-1}$, a factor $\sim 2$ higher than that found by Bolton et al. (2006) for J1011+0143. The Ly $\alpha$ luminosity is close to $L^{*}$ of the luminosity function of highredshift Ly $\alpha$ emitters (see e.g., Sobral et al. 2018).

We thank Mario Radovich, Simona Paiano, and Aldo Treves for useful discussions. This work is based on observations made with the GTC telescope, in the Spanish Observatorio del Roque de los Muchachos of the Instituto de Astrofisica de Canarias, under Director's Discretionary Time. It is also based on observations taken by the RELICS Treasury Program (GO 14096) with the NASA/ESA HST, which is operated by the Association of Universities for Research in Astronomy, Inc., under NASA contract NAS5-26555.

Facilities: GTC(OSIRIS), HST(ACS), HST(WFC2).

\section{ORCID iDs}

Daniela Bettoni (i) https://orcid.org/0000-0002-4158-6496 Renato Falomo (i) https://orcid.org/0000-0003-4137-6541 Riccardo Scarpa (1) https://orcid.org/0000-0001-9118-8739 Romano L. M. Corradi (1) https://orcid.org/0000-00027865-6056

Benedetta Vulcani ๑1 https://orcid.org/0000-0003-0980-1499

\section{References}

Agnello, A., Schechter, P. L., Morgan, N. D., et al. 2018, MNRAS, 475, 2086 Bolton, A. S., Moustakas, L. A., Stern, D., et al. 2006, ApJL, 646, L45 Bonvin, V., Courbin, F., Suyu, S. H., et al. 2017, MNRAS, 465, 4914 Cappellari, M., \& Emsellem, E. 2004, PASP, 116, 138 
Cepa, J., Aguiar-Gonzalez, M., Bland-Hawthorn, J., et al. 2003, Proc. SPIE, 4841, 1739

Cerny, C., Sharon, K., Andrade-Santos, F., et al. 2018, ApJ, 859, 159

Enia, A., Negrello, M., Gurwell, M., et al. 2018, MNRAS, 475, 3467

Foreman-Mackey, D., Hogg, D. W., Lang, D., \& Goodman, J. 2013, PASP, 125,306

Jacoby, G. H., Hunter, D. A., \& Christian, C. A. 1984, ApJS, 56, 257

Jones, T., Stark, D. P., \& Ellis, R. S. 2012, ApJ, 751, 51

Morgan, N. D., Caldwell, J. A. R., Schechter, P. L., et al. 2004, AJ, 127, 2617

Nightingale, J. W., \& Dye, S. 2015, MNRAS, 452, 2940

Oguri, M., \& Marshall, P. J. 2010, MNRAS, 405, 2579

Peng, C. Y., Ho, L. C., Impey, C. D., \& Rix, H.-W. 2002, AJ, 124, 266

Salmon, B., Coe, D., Bradley, L., et al. 2017, arXiv:1710.08930

Schechter, P. L., Morgan, N. D., Chehade, B., et al. 2017, AJ, 153, 219
Sobral, S. S., Matthee, J., Darvish, B., et al. 2018, MNRAS, 476, 4725

Suyu, S. H., Bonvin, V., Courbin, F., et al. 2017, MNRAS, 468, 2590

Suyu, S. H., Marshall, P. J., Hobson, M. P., \& Blandford, R. D. 2006 MNRAS, 371, 983

Suyu, S. H., Treu, T., Hilbert, S., et al. 2014, ApJL, 788, L35

Treu, T. 2010, ARA\&A, 48, 87

Treu, T., \& Koopmans, L. V. E. 2004, ApJ, 611, 739

Treu, T., \& Koopmans, L. V. E. 2002, MNRAS, 337, 2006

Vazdekis, A., Ricciardelli, E., Cenarro, A. J., et al. 2012, MNRAS, 424, 157

Warren, S. J., \& Dye, S. 2003, MNRAS, 590, 673

Williams, P., Agnello, A., \& Treu, T. 2017, MNRAS, 466, 3088

Williams, P. R., Agnello, A., Treu, T., et al. 2018, MNRAS, 477, L70

Wisotzki, L., Schechter, P. L., Bradt, H. V., Heinmuller, J., \& Reimers, D. 2002, A\&A, 395, 17 